\title{
Nano-Layering adds strength to the adhesive interface
}

Kumiko YOSHIHARA ${ }^{1,2}$, Noriyuki NAGAOKA ${ }^{3}$, Akiko NAKAMURA 4 , Toru HARA ${ }^{4}$, Yasuhiro YOSHIDA ${ }^{5}$, Bart VAN MEERBEEK ${ }^{6}$

1. National Institute of Advanced Industrial Science and Technology (AIST), Health Research Institute, 2217-14 Hayashi-cho, Takamatsu 761-0395, Japan

2. Okayama University, Graduate School of Medicine, Dentistry and Pharmaceutical Sciences, Department of Pathology and Experimental Medicine, 2-5-1 Shikata-cho, Kita-ku, Okayama 7008558, Japan

3. Okayama University Dental School, Advanced Research Center for Oral and Craniofacial Sciences, 2-5-1 Shikata-cho, Kita-ku, Okayama 700-8558, Japan

4. National Institute for Materials Science (NIMS), 1-2-1 Sengen, Tsukuba, Ibaraki 305-0047, Japan

5. Hokkaido University, Faculty of Dental Medicine, Department of Biomaterials and Bioengineering, Kita 13, Nishi 7, Sapporo, Hokkaido, Japan

6. KU Leuven (University of Leuven), Department of Oral Health Sciences, BIOMAT \& UZ Leuven (University Hospitals Leuven), Dentistry, Kapucijnenvoer 7, 3000 Leuven, Belgium

Keywords: Adhesives; Dentin; Electron microscopy; Hydroxyapatite; Ultrastructure 


\begin{abstract}
:
X-ray diffraction (XRD) surface analysis and ultrastructural interfacial characterization using transmission electron microscopy (TEM) confirmed that the functional monomer 10methacryloyloxydecyl dihydrogen phosphate (10-MDP) self-assembles into nano-layers at adhesive-tooth interfaces. Self-assembled nano-layering is thought to contribute to the durability of bonding to tooth dentin, although this has not been proven yet. In order to disclose this potential bond-durability contribution of nano-layering, we observed the 3D-spreading of nano-layering by a series of focused-ion-beam (FIB)-milled cross-sections by scanning electron microscopy (FIB-SEM) and examined the mechanical properties of self-assembled nano-layering using scanning probe microscopy (SPM). A commercial 10-MDP-containing two-step self-etch adhesive partially demineralized dentin up to submicron depth, forming a submicron hydroxyapatite-rich hybrid layer. TEM chemically and ultra-structurally confirmed the formation of interfacial nano-layering. FIB-SEM 3D-reconstructions disclosed a 3D-network of selfassembled nano-layering extending from the hybrid layer up to within the adjacent adhesiveresin layer. SPM revealed that nano-layering within the adhesive-resin layer possessed a higher elastic modulus than that of the surrounding adhesive resin, hereby suggesting that nanolayering contributes to the mechanical strength of adhesives like filler particles do. Nanolayering's three-dimensionally expanded structure is expected to strengthen the surrounding resin, as well to better interconnect the adhesive-resin layer to the hybrid layer. In conclusion, this exploratory study demonstrated that nano-layering constitutes a strong phase at the adhesive interface, which may contribute to the clinical longevity of the 10-MDP-based bond to dentin.
\end{abstract}




\section{Introduction}

Self-etch adhesives are user-friendly, not requiring separate etching, as they contain acidic functional monomers that simultaneously 'condition' and 'prime' tooth tissue. Key constituents of self-etch adhesives are functional monomers (Van Meerbeek et al., 2020), which directly determine interfacial ultrastructure and chemical interaction with direct impact on bond durability (Yoshihara et al., 2010, 2019). The adhesion-decalcification (AD) concept mechanistically explained how acidic molecules chemically interact with HAp-based tissue following either an adhesion or decalcifation route (Van Meerbeek et al., 2020). Acidic functional monomers produce hybrid layers with different ultrastructure depending on the dissolution rate of their Ca-salts. In brief, acidic monomers with soluble Ca-salts produce thicker HAp-poor/free hybrid layers at dentin with abundant collagen exposure, as representative for etch-and-rinse adhesives. Contrastingly, functional monomers producing stable Ca-salts partially/shallowly demineralize dentin, resulting in submicron hybrid layers with collagen remained protected by HAp, as representative for mild self-etch adhesives. Besides providing micromechanical interlocking through etching, additional primary chemical interaction of functional monomers with HAp stabilizes the adhesive interface. In particular, 10-MDP revealed intense and stable chemical bonding efficacy, which was later also documented for some fluoro-carbon analogues (Yoshihara et al., 2014). In continued research, Yoshihara et al. (2019) discovered that 10-MDP self-assembled into about 4-nm nano-layers, consisting of two sublayers of parallel oriented 10MDP monomers in opposite direction with one nano-layer connected to the next one via Ca that formed a stable ionic bridge connecting the phosphate groups of adjacent 10-MDP's. This theoretical molecular structural model of nano-layering was proposed based on X-ray diffraction (XRD) data (Yoshida et al., 2012). Nano-layering at adhesive interfaces was next visualized using transmission electron microscopy (TEM) by Yoshihara et al. (2010). The mechanisms of interfacial nano-layering were characterized ultra-morphologically and chemically, collecting data from specimens prepared in a similar manner as done clinically, this correlatively using scanning TEM with energy-dispersive X-ray spectrometry (STEM-EDS), XRD and solid-state nuclear magnetic resonance (NMR). Most recently, water-stable 10-MDP_Ca salts were found to consist of the very stable $\mathrm{CaRPO}_{4}$, meaning that the two OH-groups of 10-MDP's phosphate group ionically reacted with Ca (Yoshihara et al., 2019).

Hybrid layers have abundantly been documented to degrade with time (Breschi et al., 2018). Immediate and aged micro-tensile bond-strength tests revealed the hybrid layer to fracture as being the most degradation-sensitive part of the adhesive assembly (Frassetto et al., 2016). Many researchers attempted to strengthen the hybrid layer, for example by optimizing the adhesive's composition, improving its degree of conversion, or by adding novel agents such as collagen crosslinkers, all of which having been investigated amply (Spencer et al., 2014; Frassetto 
et al., 2016).

Until the date of this paper, no mechanical property data of 10-MDP_Ca-salt nano-layering are available in light of its potential contribution to adhesive interfacial stability. In this study, we therefore mapped elastic modulus across cross-sectioned interfaces to assess the mechanical properties of self-assembled nano-layering using scanning probe microscopy (SPM). We also observed the 3D-spreading of nano-layering by reconstructing a series of focused-ion-beam (FIB) milled adhesive-dentin cross-sections imaged by scanning electron microscopy (FIB-SEM). The null hypothesis tested was that no difference in EM was measured between 10-MDP_Ca-salt nano-layering and the surrounding adhesive resin.

\section{Materials and Methods}

\section{Specimen preparation for FIB-SEM, TEM and SPM}

The occlusal crown of four extracted non-carious human third molars (approved by the Commission for Medical Ethics of Okayama University Hospital under file number 1606-020) was removed using a diamond saw (Accutom 50, Struers, Ballerup, Denmark), upon which the exposed dentin was wet-ground using 600-grit SiC paper (WTCC-S, Nihonkenshi, Fukuyama, Japan) to prepare a flat and uniform smear layer, enabling good visualization of interfacial ultrastructural detail. The 10-MDP-containing two-step self-etch adhesive Clearfil SE Bond 2 ('CSE2'; Kuraray Noritake) was applied on dentin according to the manufacturer's instructions, involving application of C-SE2 primer using a microbrush, followed by air-blowing at 20 sec after application. Next, an experimental adhesive resin with the same composition as that of C-SE2 but without filler (Kuraray Noritake) was applied, so to avoid filler interference with the SPM mapping of EM across the adhesive interface. The adhesive resin was cured for 10 s using the LED light-curing unit G-light Prima II (GC, Tokyo, Japan). Then, the low-viscosity composite resin Clearfil Protect Liner F (Kuraray Noritake) was applied and light-cured. Upon 24-h water storage, the adhesive-treated dentin specimens were mid-sectioned in two halves, upon which per specimen half up to five about $1 \times 1-\mathrm{mm}$ rectangular slabs were cut. All slabs were successively fixed in glutaraldehyde (Nacalai Tesque, Kyoto, Japan), gradually dehydrated in ethanol and embedded in epoxy resin (TAAB Laboratories Equipment, Aldermaston, UK). Further specimen preparation/processing for FIB-SEM, TEM and SPM is detailed below.

\section{FIB-SEM}

Eight slabs embedded in epoxy resin with the cross-sectioned adhesive-dentin interface exposed at the surface were mechanically polished using diamond lapping films (3M, St.Paul, MN, USA) and argon-ion polishing (SM-090101 Cross-Section Polisher, JEOL, Tokyo, Japan). Orthogonal FIB- 
SEM (SMF-1000, HITACHI, Tokyo, Japan) imaging was conducted for 3D-ultrastructural characterization. The FIB and SEM ion/electron beams were orthogonally aligned, instead of being set at the standard angle of $60^{\circ}$, to obtain high spatial resolution and high-contrast SEM photomicrographs (Wirth, 2009). Lucis Pro (Microtechnics, Granite Bay, CA, USA) and Stacker and Visualizer-Kai (System In Frontier, Tokyo, Japan) software packages were utilized for 3D reconstruction. SEM was performed at $0.5 \mathrm{kV}$. Images were obtained using an annular in-lens secondary electron detector and an annular in-lens energy-selected backscattered electron detector. The observed area was $10 \times 10 \mu \mathrm{m}$ with $10 \mathrm{~nm} /$ pixels each. Serial sectioning observations were carried out with a slice pitch of $10 \mathrm{~nm}$ for 990 sheets. The obtained images were rendered by removing resin and pseudo-colored according contrast.

\section{TEM}

From eight slabs, originating from four teeth, numerous ultra-thin adhesive-dentin crosssections were cut using routine ultra-microtomy (LEICA EM UC6, Leica, Vienna, Austria), prior to being observed by TEM at $200 \mathrm{kV}$, utilizing a JEM-2100 microscope (JEOL).

\section{Quantitative mechanical property mapping by SPM}

Similar to the FIB-SEM specimen-preparation procedure, eight embedded specimens were mechanically polished using diamond lapping films (3M) and argon-ion polished (SM-090101 Cross-Section Polisher, JEOL). The SPM system employed was a Bruker MultiMode 8 SPM equipped with a Nanoscope $V$ controller and Nanoscope analysis software (Bruker Nano Surface Business, Santa Barbara, CA, USA). Cross-sectioned surfaces were quantitatively characterized using the PeakForce QNM (Quantitative NanoMechanics) SPM module under ambient conditions. Elastic modulus (EM) was calculated by NanoScope analysis software using the Derjaguin-Muller-Toropov (DMT) model (Derjaguin et al., 1975; Wang et al., 2013). Samples were scanned using a probe (RTESPA-525, Bruker) with a nominal radius of $8 \mathrm{~nm}$ and a nominal spring constant of $200 \mathrm{Nm}^{-1}$ with $0.501 \mathrm{~Hz}$ scan rates, a 250-mV amplitude set point and a 150-nm peak force amplitude. After SPM, the samples were observed by SEM (JSM-6701F, JEOL).

\section{S/TEM observation of a clinically C-SE treated tooth}

A tooth extracted because of periodontal pathology (approved by the Commission for Medical Ethics of Okayama University Hospital under file number 1901-036) was collected. The tooth was adhesively restored 9 years ago using Clearfil SE Bond ('C-SE' as pre-cursor of C-SE2; Kuraray Noritake) and the resin-based composite Clearfil AP-X (Kuraray Noritake). The tooth was fixed in glutaraldehyde, gradually dehydrated in ethanol and embedded in epoxy resin. After the 
adhesive-dentin interface was exposed, the interface was mechanically polished using diamond lapping film (3M, St. Louis, MO, USA), followed by broad argon-ion-beam polishing using a crosssection polisher (SM-09020 CP, JEOL). Subsequently, a thin layer of carbon was deposited on the surface (JEE-420T, JEOL), upon which the specimens were examined with Feg-SEM (JSM-6701F, $\mathrm{JEOL}$ ) operated at $5 \mathrm{kV}$ and employing an annular semiconductor detector. For TEM, ultrathin sections were cut (Leica EM UC7, Leica, Vienna, Austria), prior to being examined with highresolution 200-kV TEM utilizing a JEM-2100 (JEOL) microscope.

\section{Results}

FIB-SEM

FIB-SEM 3D-reconstruction resulted in a $10-\mu \mathrm{m}^{3}$ block of the mild self-etch adhesive-dentin interface, revealing a 500-nm to 1- $\mu \mathrm{m}$ thick HAp-rich hybrid layer with abundant nano-layering produced on top (Fig. 1a). 10-MDP_Ca salts were detected within the hybrid layer and widely spread along the interface within the adhesive-resin area. By adding pseudocolor in Fig. $1 \mathrm{~b}$ and digitally removing resin in Fig. 1c, 10-MDP_Ca salt nano-layering was observed more clearly. Within the composite resin, filler particles were visible.

\section{TEM}

TEM of the interface of C-SE2 bonded onto dentin revealed a 0.5-0.7 $\mu \mathrm{m}$ thick hybrid layer, in which HAp was found to have remained without hardly any collagen exposure (Fig. 2b). At the hybrid-layer top, regular longitudinally layered and often curved structures were observed. At higher magnification, these layers represented nano-layers with a periodicity of approximately $3.5 \mathrm{~nm}$ (Fig. 2c).

\section{SPM}

DMT-EM mapping clearly indicated differences in surface brightness with the brighter regions representing areas exhibiting higher EM, corresponding to stiffer phases, versus lower EM at the darker regions, corresponding to softer phases (Fig. 3). Across the adhesive-dentin interface, EM varied from 1.1 to $10.2 \mathrm{GPa}$. As mineralized tissue, unaffected dentin underneath the interface appeared brightest, with the highly mineralized peritubular dentin exhibiting clearly higher EM than intertubular dentin. The upper area showed the low-viscosity composite resin, clearly indicating the higher brightness and thus higher EM of the contained silica filler. The surrounding resin matrix was darker, representing a softer (less stiff) surface, as was also detected for the unfilled adhesive layer between low-viscosity composite resin and hybrid layer, for which similar EMs were recorded. High magnification showed a darker (lower EM) hybrid layer than dentin, but was brighter (higher EM) than the adhesive resin. Characteristic brighter extensions within 
the adhesive resin, originating at the hybrid layer, represent the formed nano-layers that were identified as regions with higher EM than that of the surrounding resin within the adhesive-resin layer.

\section{SEM}

Upon SPM, the same FIB-sectioned specimens were observed by SEM (Fig. 4). Low magnification revealed a $10-\mu \mathrm{m}$ thick adhesive-resin layer between the low-viscosity composite resin and dentin. At high magnification, abundant nano-layering extending from the hybrid layer was detected and fully corresponded to that disclosed by SPM.

\section{S/TEM observation of a clinically C-SE treated tooth}

The clinical case of an adhesively restored tooth extracted after 9 years of clinical service revealed light-microscopy (LM) photographs of the exposed composite-tooth interface (Fig. 5). SEM disclosed a tight composite-adhesive-dentin interface, while the adhesive and composite resin closest to dentin contained several voids, which should be attributed to de-bonded silica filler in the adhesive layer. TEM revealed a 500-nm thick hybrid layer. High-magnification TEM clearly disclosed nano-layering close to the hybrid layer, which was detected after 9-year in-vivo service.

\section{Discussion}

The study's aim was to measure the mechanical strength of nano-layering in potential contribution to adhesive interfacial stability. Considering its nanometer size and regionally varying occurrence, measurement of mechanical properties of nanometer-sized interfacial structures is challenging. SPM methods have been developed to detect interactions of the cantilever tip with the sample surface; among diverse applications, they have enabled measuring mechanical properties such as EM on the micrometer to subnanometer scale. When adhesivedentin interfaces were mapped using the DMT-EM SPM module, the self-assembled nanolayering of 10-MDP_Ca salts presented with higher EM than the surrounding adhesive resin. Additionally, this study imaged nano-layering distribution along the adhesive-dentin interface in 3D using 3D-reconstruction of consecutive FIB cross-sections. Full nano-layering identification was obtained by complementing XRD chemical surface analysis with TEM/SEM ultra-structural interfacial analysis.

Having been recorded as most effective functional monomer, 10-MDP is often the main functional monomer contained in the newest generation of universal adhesives (Van Meerbeek et al., 2020). 10-MDP has a characteristic molecular structure with a long spacer group ending in a polymerizable methacrylate group, well separated from the phosphate group at the other 
monomer's end with chemical binding potential to Ca of HAp (Yoshihara et al., 2018). 10-MDP interacts with dentin in three ways (Yoshihara et al., 2019): (1) achieving shallow surface microretention by etching without fully uncovering collagen; (2) binding electrostatically to calcium of HAp remaining around collagen; and (3) self-assembling into nanometer-sized structures consisting of 10-MDP_Ca salts, known as nano-layering (Yoshihara et al., 2010). Nano-layering is confirmed by XRD, revealing three characteristic peaks representing the regularly ordened 10 MDP_Ca salts. Based on the molecular structure of 10-MDP, 10-MDP analogues with nanolayering potential were synthesized and appeared as effective as 10-MDP with some even possessing better hydrolytic resistance (Yoshihara et al., 2014).

Much adhesive dentistry-related research involves morphologic examination of hybrid-layer integrity, often to study bond-degradation mechanisms (Frassetto et al., 2016). Mechanical properties of adhesive interfaces are most often quantitatively assessed in terms of bond strength measured in different modes (De Munck et al., 2012). Basically, the overall strength of the complete adhesive-dentin assembly is measured, commonly also influenced by the involved tooth substrate and restorative material. In order to evaluate interface quality, nano-identation has been employed to measure nano-hardness and nano-EM across adhesive interfaces (Van Meerbeek et al., 1993). However, this technique is limited regarding indent size/depth, being often too large/deep, by which mixed substrate/material property data are collectively recorded. New opportunities arose with the introduction of atomic force microscopy (AFM) equipped with nano-indentation. AFM-cantilever tips have a radius of approximately $20 \mathrm{~nm}$, although reaching nano-level resolution using AFM nano-indentation remains difficult, mainly in case of highly varying surface topography (Schulze et al., 2005). Pioneering AFM research was conducted to measure in-situ mechanical properties of fully hydrated calcified tissues and across the dentinoenamel junction using AFM-based nano-indentation (Kinney et al., 1996; Marshall et al., 2001). Likewise, AFM nano-indentation was used to determine hardness and EM across adhesive-dentin interfaces and of hybrid layers in particular (Oliveira et al., 2004, Schulze et al., 2005). As appeared for this AFM nano-indentation (Ho et al., 2004), most challenging for SPM is the need to prepare artifact- and smear-free, and nearly completely flat cross-sections. Metallographical grinding and polishing of interface specimens cannot prevent polishing debris be smeared across the interface and damage the softer collagen fibrils within the hybrid layer (Yoshida et al., 1999; Ho et al., 2004). Ultra-microtomy sectioning with a diamond knife was therefore used to prepare clean adhesive interfaces, enabling AFM-imaging of hybrid-layer collagen (Van Meerbeek et al., 1999; Yoshida et al., 1999). However, due to differences in surface properties, the interface specimens revealed a clear dip at the adhesive-resin layer in between the harder dentin substrate and particle-filled composite resin. In a prior pilot study, metallographical polishing and ultra-microtomy were found inapplicable to assess nano-layering 
with SPM, as both methods induced surface distortions and scratches. We next prepared interfacial cross-sections using broad argon-ion-beam cross-section polishing, which resulted in much flatter surfaces with minimum damage and distortion (Erdman et al., 2006). Such gentle argon-ion-beam cross-sectioning appeared effective to assess mechanical properties across adhesive-tooth assemblies that consist of different materials/substrates with varying properties, as there are unaffected dentin, the hybrid and adhesive-resin layers, and the composite resin.

10-MDP_Ca-salts are stable and insoluble in water (Yoshida et al., 2012), with 10-MDP_Casalt nano-layering outgrowing from the hybrid layer in a hairy fashion into the adhesive-resin layer (Yoshihara et al., 2019). In this way, the adhesive interface can be regarded as a functionally graded material (Marshall et al., 2001), often being referred to as FGM, with properties such as stiffness (EM) to gradually change from the mineralized unaffected dentin across the partially demineralized hybrid layer towards the adhesive resin, this to the direct benefit of producing a stress-withstanding/absorbing adhesive interface. The formed nano-layering, consisting of two rows of regularly aligned 10-MDP molecules, with one nano-layer strongly connected by stable Ca-salt formation to the next nano-layer in a 3D perspective, further optimizes this functional gradient at the adhesive-dentin interface, since the nano-layered structure appeared in this study to possess mechanical properties in between that of the hybrid and adhesive-resin layer.

The actual contribution of nano-layering to adhesive interfacial stability however remained unknown up to the publication date of this work. This knowledge is nevertheless of high interest, for instance to know if interfacial nano-layering intentionally should be intensified to make adhesive interfaces more durable. Anecdotal clinical evidence of nano-layering stability is provided by a clinical case with nano-layering still detected after 9-year clinical service at the adhesive-dentin interface of a composite restoration bonded to dentin using Clearfil SE Bond (Kuraray Noritake), being the commercial precursor of the self-etch adhesive used in this study (Fig. 5).

SPM, or atomic force microscopy (AFM), enables analysis at atomic level. Among several SPM measurement methods, PeakForce Quantitative Nanomechanical Property Mapping (QNM) probes surface topography and mechanical properties at the same time by making a forcedistance curve at each spot and fitting this force-distance curve with the DMT model of elastic contact (Drijkoningen et al., 2014). SPM in DMT mode revealed higher EM for dentin, attributable to HAp, and for the composite-resin filler consisting of silica glass. The intermediary EM mapped at the submicron hybrid layer must be ascribed to the solely partial dentin demineralization with HAp still surrounding collagen. EM of the adhesive resin was lower as compared to that of dentin and the low-viscosity composite resin. However, EM of nano-layers surpassed that of the adhesive resin, by which the null hypothesis that no difference in EM was measured between 10-MDP_Ca-salt nano-layering and the surrounding adhesive, failed to be 
accepted. This higher EM of nano-layers should be attributed to the compactly ordened structure of interconnected 10-MDP_Ca-salts. Having better mechanical properties than those of the surrounding adhesive resin, these nano-layers are expected to contribute to the stability of the adhesive-dentin interface. EM mapping of adhesive-dentin interfaces by SPM may be useful to study bond-degradation processes when adhesive interfaces are exposed to accelerated aging regimes.

Dental adhesives often contain filler particles in order to increase viscosity, to provide radiopacity, to improve the adhesive's mechanical properties and to act as film-thickness regulator with shock-absorbing potential (Lohbauer et al., 2010; Van Meerbeek et al., 2020). Adding filler to adhesives resulted in different effects on their mechanical properties, while these depends also on the type of filler and their composition, but also on the test method applied. Optimized micro/nano-silica filler content in adhesives was shown to improve bond strength (Miyazaki et al., 1995; Kim et al., 2005; Lohbauer et al., 2010). TEM confirmed that filler particles hardly infiltrate hybrid layers (Tay et al., 1999), while they can penetrate with resin within opened dentin tubules, strengthening resin tags (Frankenberger et al., 2001; Lohbauer et al., 2010). Otherwise, Miyazaki et al. (1995) revealed that higher amounts of silica filler reduced the degree of conversion and resulted in lower bond strength. Others found that 1-10\% filler increased the adhesive's flexural (Kim et al., 2005) and cohesive strength (Conde et al., 2009). The formed nano-layers could be considered as self-formed filler, promoting the adhesive resin's strength. Moreover, while micro/nano-filler particles possess a spheric structure, 10-MDP_Ca salts selfassembled into a three-dimensional structure that grew and expanded on the hybrid-layer top into the adhesive-resin layer, hereby strengthening both the hybrid and adhesive-resin layer, as well as their interconnection.

In conclusion, this study found that 10-MDP_Ca-salt nano-layers possess higher EM than the surrounding adhesive resin. Their presence within the hybrid layer extending into the overlying adhesive resin strengthens both the hybrid and adhesive-resin layer. Their 3D-continuous structure results in enhanced strengthening as compared to filler, while it may also more tightly interconnect the hybrid layer with the adhesive-resin layer. While in-vivo case evidence of nanolayer stability exists, further research should test the nano-layering's resistance against hydrolytic degradation.

\section{Author Contributions}

Yoshihara K, contributed to conception, design, data acquisition, analysis and interpretation, drafted and critically revised the manuscript; Nagaoka $N$, contributed to conception, design, data acquisition, analysis and interpretation, and critically revised the manuscript; Nakamura A, contributed to conception, design, data acquisition, analysis and critically revised the 
manuscript; Hara T, contributed to conception, design, data acquisition, analysis and critically revised the manuscript; Yoshida $Y$, contribute to conception, interpretation, and critically revised the manuscript; Van Meerbeek B, contribute conception, interpretation, drafted and critically revised the manuscript. All authors gave final approval and agree to be accountable for all aspects of the work.

\section{Acknowledgements}

This work was supported by the NIMS microstructural characterization platform as a program of "Nanotechnology Platform" of the Ministry of Education, Culture, Sports, Science and Technology (MEXT) of Japan. This study was supported by JSPS KAKENHI with the grant number JP 18K17068. The authors declare that they have no conflict of interest in this research. We thank Kuraray Noritake for providing the experimental adhesive formulation. 


\section{References}

Breschi L, Maravic T, Cunha SR, Comba A, Cadenaro M, Tjäderhane L, Pashley DH, Tay FR, Mazzoni A. 2018. Dentin bonding systems: From dentin collagen structure to bond preservation and clinical applications. Dent Mater. 34(1):78-96.

Conde MC, Zanchi CH, Rodrigues-Junior SA, Carreño NL, Ogliari FA, Piva E. 2009. Nanofiller loading level: Influence on selected properties of an adhesive resin. J Dent. 37(5):331-335.

De Munck J, Mine A, Poitevin A, Van Ende A, Cardoso MV, Van Landuyt KL, Peumans M, Van Meerbeek B. 2012. Meta-analytical review of parameters involved in dentin bonding. J Dent Res. 91(4):351-7.

Derjaguin BV, Muller VM, Toporov YP. 1975. Effect of contact deformations on the adhesion of particles. J. Colloid Interface Sci. 53(2):314-326.

Drijkoningen J, Kesters J, Vangerven T, Bourgeois E, Lutsen L, Vanderzande D, Maes W, D'Haen J, Manca J. 2014. Investigating the role of efficiency enhancing interlayers for bulk heterojunction solar cells by scanning probe microscopy. Org Electron. 15(6):1282-1289.

Erdman N, Campbell R, Asahina S. 2006. Precise SEM Cross Section Polishing via Argon Beam Milling. Micros Today. 14(3):22-25.

Frankenberger R, Perdigão J, Rosa BT, Lopes M. 2001. "No-bottle" vs "multi-bottle" dentin adhesives - a microtensile bond strength and morphological study. Dent Mater. 17(5):373380.

Frassetto A, Breschi L, Turco G, Marchesi G, Di Lenarda R, Tay FR, Pashley DH, Cadenaro M. 2016. Mechanisms of degradation of the hybrid layer in adhesive dentistry and therapeutic agents to improve bond durability - A literature review. Dent Mater. 32(2):e41-53.

Ho SP, Goodis H, Balooch M, Nonomura G, Marshall SJ, Marshall G. 2004. The effect of sample preparation technique on determination of structure and nanomechanical properties of human cementum hard tissue. Biomaterials. 25(19):4847-57.

Kim JS, Cho BH, Lee IB, Um CM, Lim BS, Oh MH, Chang CG, Son HH. 2005. Effect of the hydrophilic nanofiller loading on the mechanical properties and the microtensile bond strength of an ethanol-based one-bottle dentin adhesive. J Biomed Mater Res B Appl Biomater. 72(2):284291.

Kinney JH, Balooch M, Marshall SJ, Marshall GW Jr, Weihs TP. 1996. Atomic force microscope measurements of the hardness and elasticity of peritubular and intertubular human dentin. J Biomech Eng. 118(1):133-135.

Lohbauer U, Wagner A, Belli R, Stoetzel C, Hilpert A, Kurland HD, Grabow J, Müller FA. 2010. Zirconia nanoparticles prepared by laser vaporization as fillers for dental adhesives. Acta Biomater. 6(12):4539-4546.

Marshall GW Jr, Balooch M, Gallagher RR, Gansky SA, Marshall SJ. 2001. Mechanical properties 
of the dentinoenamel junction: AFM studies of nanohardness, elastic modulus, and fracture. J Biomed Mater Res. 54(1):87-95.

Miyazaki M, Ando S, Hinoura K, Onose H, Moore BK. 1995. Influence of filler addition to bonding agents on shear bond strength to bovine dentin. Dent Mater. 11(4):234-238.

Oliveira SS, Marshall SJ, Habelitz S, Gansky SA, Wilson RS, Marshall GW. 2004. The effect of a selfetching primer on the continuous demineralization of dentin. Eur J Oral Sci. 112(4):376-383.

Tay FR, Moulding KM, Pashley DH. 1999. Distribution of nanofillers from a simplified-step adhesive in acid-conditioned dentin. J Adhes Dent. 1(2):103-117.

Schulze KA, Oliveira SA, Wilson RS, Gansky SA, Marshall GW, Marshall SJ. 2005. Effect of hydration variability on hybrid layer properties of a self-etching versus an acid-etching system. Biomaterials. 26(9):1011-1018.

Spencer P, Ye Q, Misra A, Goncalves SE, Laurence JS. 2014. Proteins, pathogens, and failure at the composite-tooth interface. J Dent Res. 93(12):1243-9.

Van Meerbeek B, Willems G, Celis JP, Roos JR, Braem M, Lambrechts P, Vanherle G. 1993. Assessment by nano-indentation of the hardness and elasticity of the resin-dentin bonding area. J Dent Res. 72(10):1434-1442.

Van Meerbeek B, Yoshida Y, Snauwaert J, Hellemans L, Lambrechts P, Vanherle G, Wakasa K, Pashley DH. 1999. Hybridization effectiveness of a two-step versus a three-step smear layer removing adhesive system examined correlatively by TEM and AFM. J Adhes Dent. 1(1):7-23.

Van Meerbeek B, Yoshihara K, Van Landuyt K, Yoshida Y, Peumans M. 2020. From Buonocore's pioneering acid-etch technique to self-adhering restoratives. A status perspective of rapidly advancing dental adhesive technology. J Adhes Dent. 22(1):7-34.

Wang D, Russell TP, Nishi T, Nakajima K. 2013. Atomic force microscopy nanomechanics visualizes molecular diffusion and microstructure at an interface. ACS Macro Letters. 2(8):757-760.

Wirth R. 2009. Focused Ion Beam (FIB) combined with SEM and TEM: Advanced analytical tools for studies of chemical composition, microstructure and crystal structure in geomaterials on a nanometer scale. Chemical Geology. 261(3-4):217-229.

Yoshida Y, Van Meerbeek B, Snauwaert J, Hellemans L, Lambrechts P, Vanherle G, Wakasa K, Pashley DH. 1999. A novel approach to AFM characterization of adhesive tooth-biomaterial interfaces. J Biomed Mater Res. 47(1):85-90.

Yoshida Y, Yoshihara K, Nagaoka N, Hayakawa S, Torii Y, Ogawa T, Osaka A, Van Meerbeek B. 2012. Self-assembled nano-layering at the adhesive interface. J Dent Res. 91(4):376-381.

Yoshihara K, Hayakawa S, Nagaoka N, Okihara T, Yoshida Y, Van Meerbeek B. 2018. Etching efficacy of self-etching functional monomers. J Dent Res. 97(9):1010-1016.

Yoshihara K, Nagaoka N, Yoshida Y, Van Meerbeek B, Hayakawa S. 2019. Atomic level observation and structural analysis of phosphoric-acid ester interaction at dentin. Acta Biomater. 97:544- 
556.

Yoshihara K, Yoshida Y, Hayakawa S, Nagaoka N, Kamenoue S, Okihara T, Ogawa T, Nakamura M,

Osaka A, Van Meerbeek B. 2014. Novel fluoro-carbon functional monomer for dental bonding. J Dent Res. 93(2):189-194.

Yoshihara K, Yoshida Y, Nagaoka N, Fukegawa D, Hayakawa S, Mine A, Nakamura M, Minagi S,

Osaka A, Suzuki K et al. 2010 Nano-controlled molecular interaction at adhesive interfaces for hard tissue reconstruction. Acta Biomater. 6(9):3573-3582.

Yoshihara K, Yoshida Y, Nagaoka N, Hayakawa S, Okihara T, De Munck J, Maruo Y, Nishigawa G, Minagi S, Osaka A et al. 2013. Adhesive interfacial interaction affected by different carbonchain monomers. Dent Mater. 29(8):888-897. 


\section{Figure captions:}

Figure $13 D$-reconstructed FIB-SEM cross-sections of a mild self-etch adhesive-dentin interface in (a), with pseudocolors added in (b) and additional digital removal of resin in (c).

Figure 2. Collage of TEM photomicrographs of the interface of Clearfil SE Bond 2 (Kuraray Noritake) bonded to dentin, imaged at different magnifications and revealing a submicron hybrid layer in and nano-layering with a periodicity of approximately $3.5 \mathrm{~nm}$.

Figure 3. Derjaguin-Muller-Toporov (DMT) elastic modulus (EM) mapping by SPM across the adhesive-dentin interface produced by Clearfil SE Bond 2 (Kuraray Noritake) bonded to dentin at different magnifications.

Figure 4. SEMs of the FIB cross-sectioned interface of Clearfil SE Bond 2 (Kuraray Noritake) bonded to dentin and imaged at the same area as scanned by SPM in Figure 3. (a) Lowmagnification photomicrograph revealed a tight adhesive-dentin interface with disclosure of the grey-colored submicron hybrid layer and nano-layering extending from the hybrid layer within the overlying adhesive resin in (b) and (c). The SEM photomicrograph in (c) was taken at about the same position and magnification as the SPM-scanned image in (d).

Figure 5. Clinical case of an extracted adhesively restored tooth, of which ultra-structural SEM and TEM interfacial characterization revealed nano-layering after 9-year clinical service at the adhesive-dentin interface of a composite restoration bonded to dentin using Clearfil SE Bond (Kuraray Noritake), being the commercial precursor of the 10-MDP-based self-etch adhesive applied in this study. 


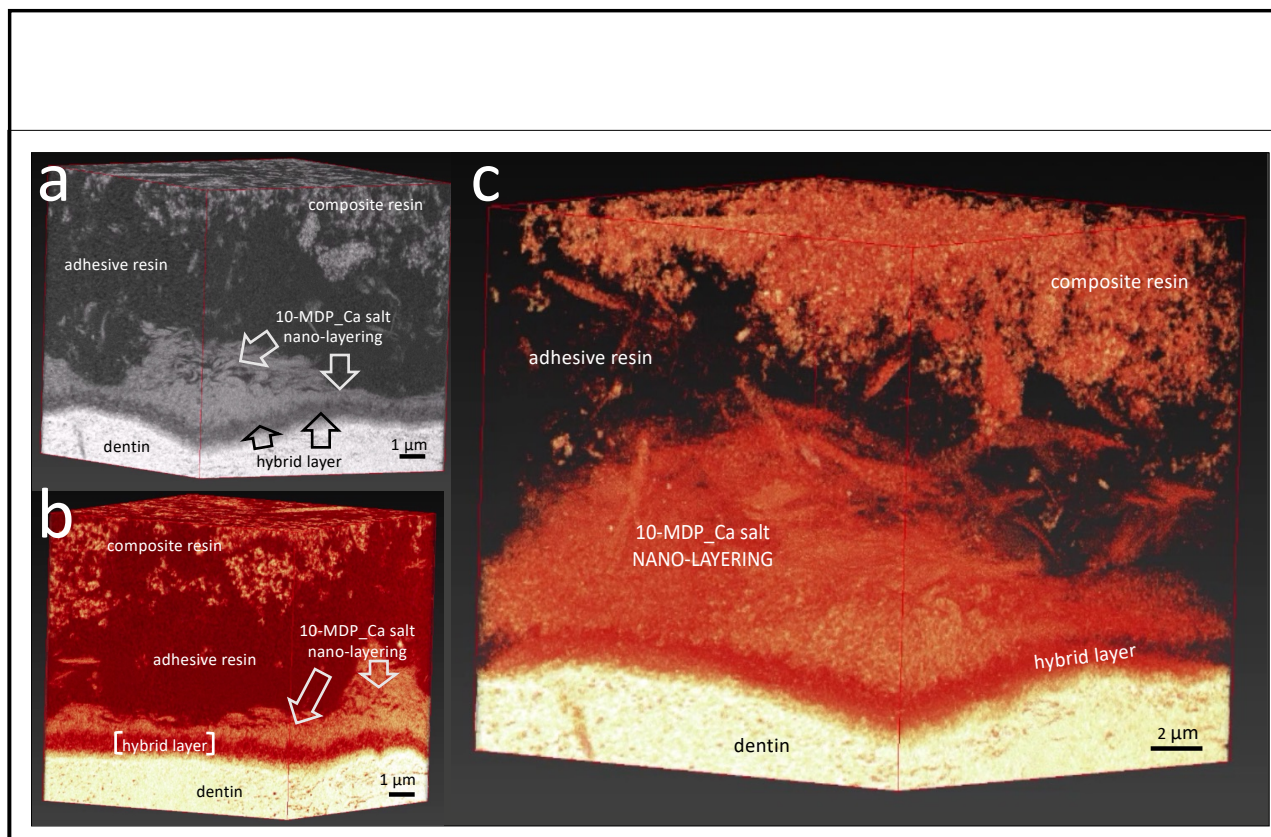

Figure 1

1

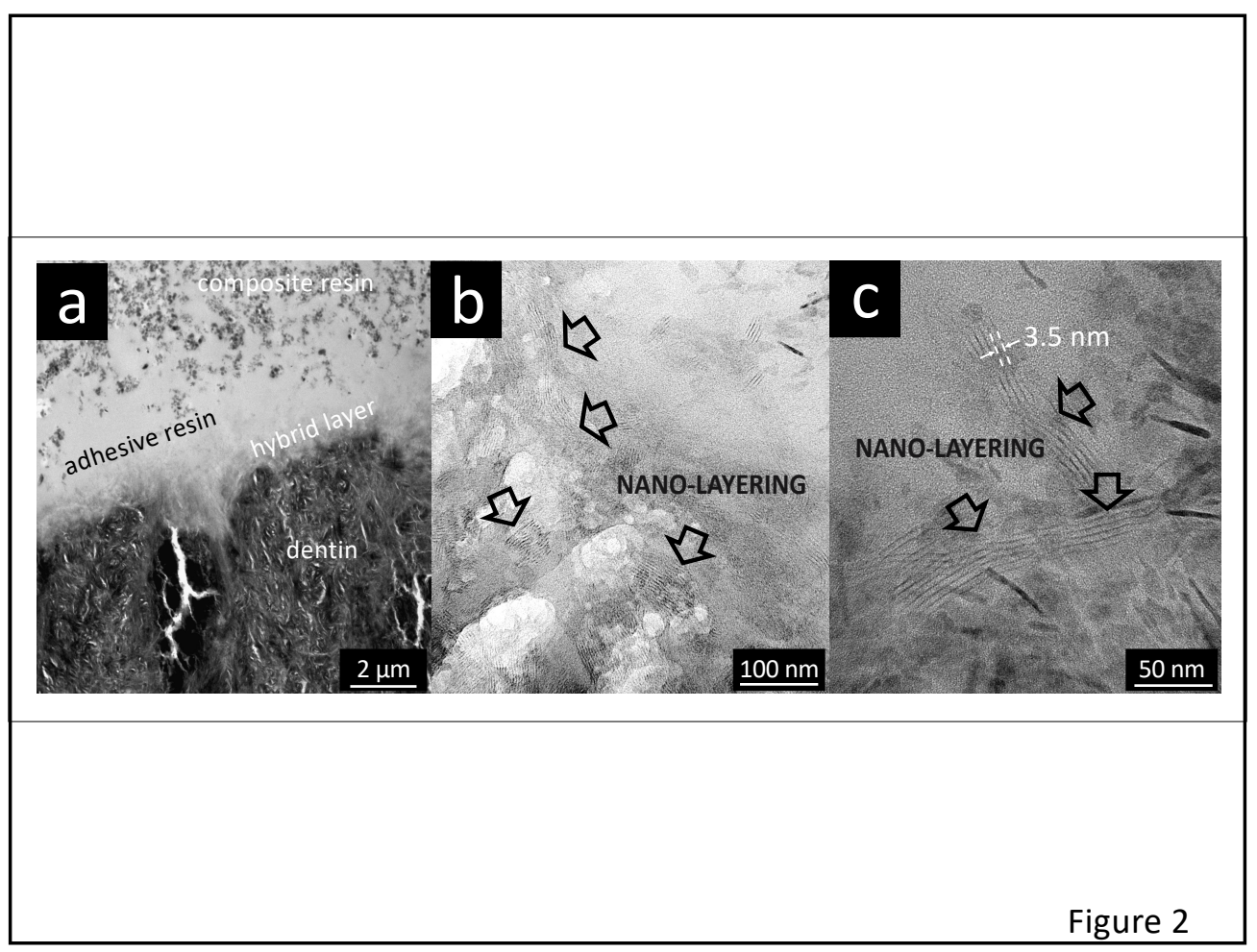

2 


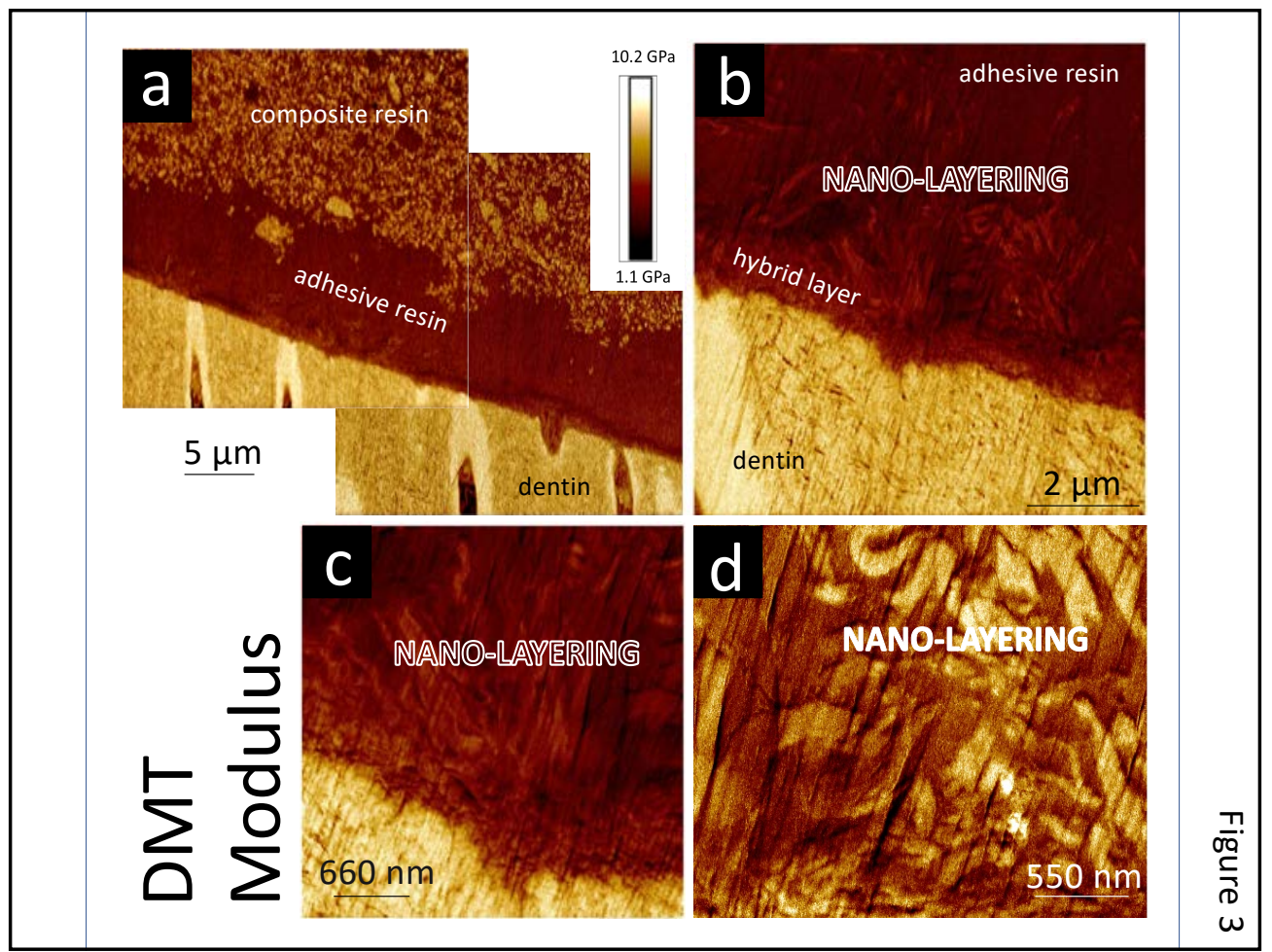

3

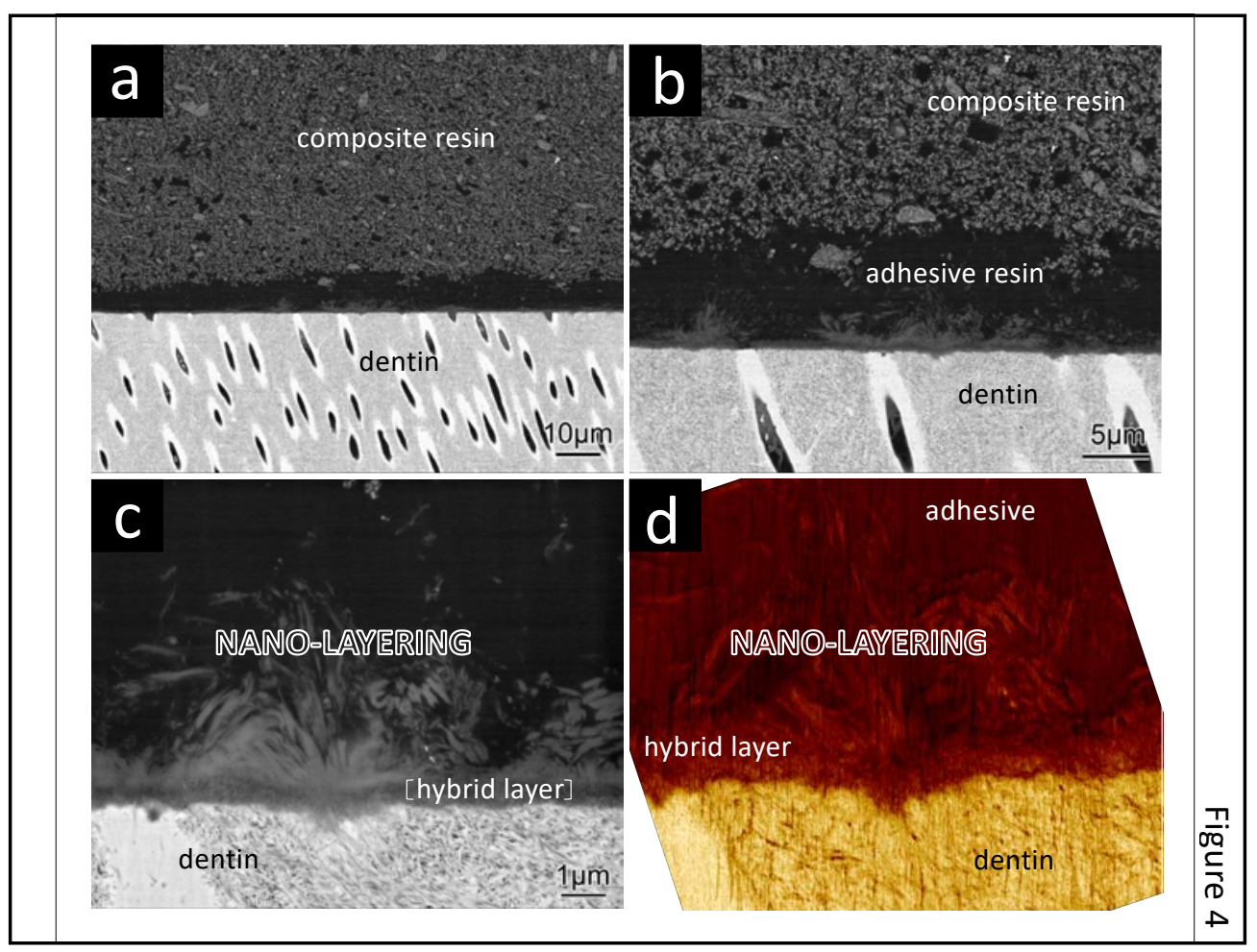




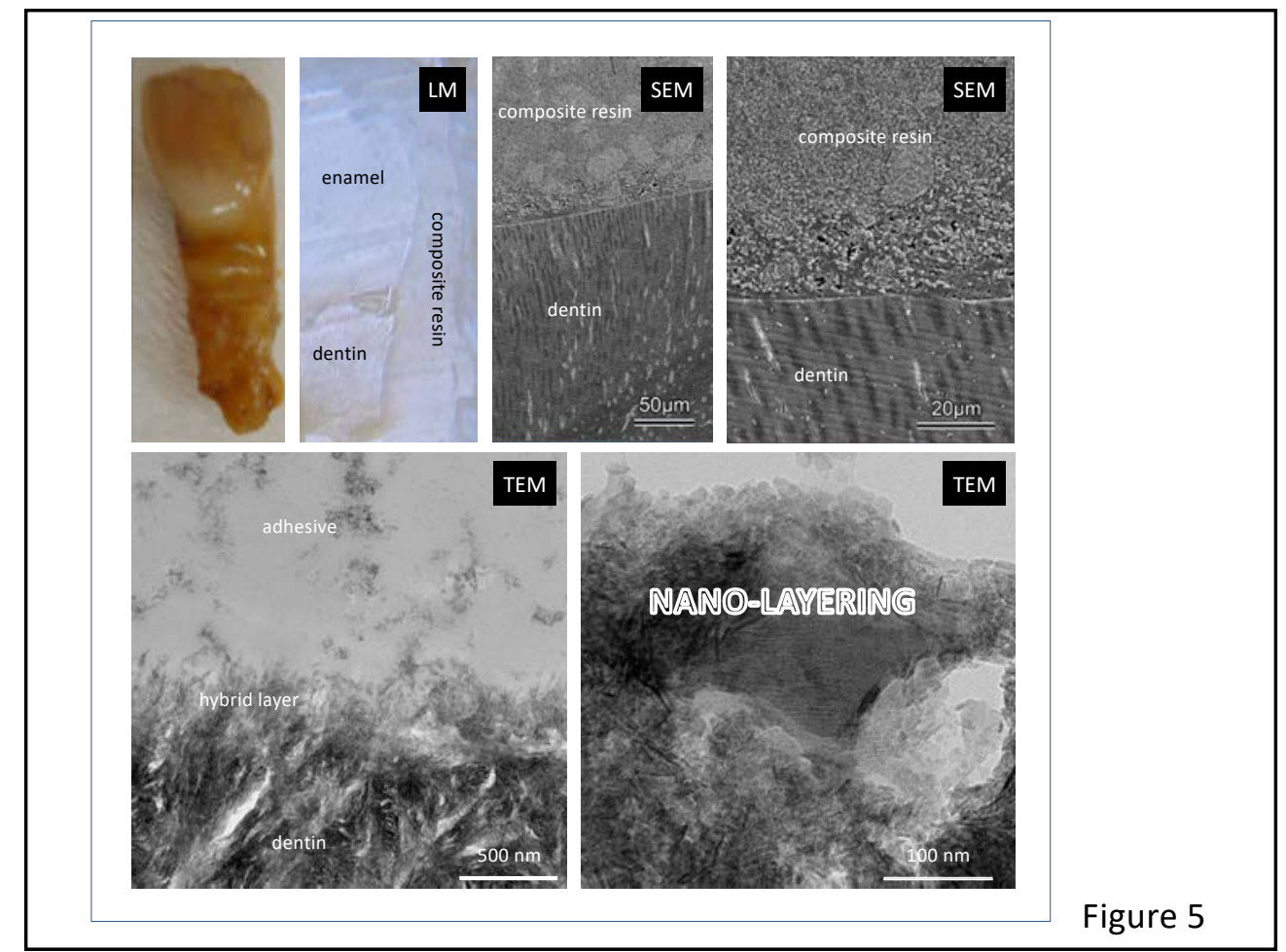

5 